\title{
Is the Mean Blood Leukocyte Telomere Length a Predictor for Sporadic Thoracic Aortic Aneurysm? Data from a Preliminary Study
}

\author{
Carmela Rita Balistreri, ${ }^{1}$ Calogera Pisano, ${ }^{2}$ Daniele Merlo, ${ }^{2}$ Khalil Fattouch, ${ }^{2}$ Marco Caruso, ${ }^{3}$ \\ Egle Incalcaterra, ${ }^{3}$ Giuseppina Colonna-Romano, Giovanni Ruvolo, ${ }^{2}$ and Giuseppina Candore ${ }^{1}$
}

\begin{abstract}
Telomeres have been postulated as a universal clock that shortens in parallel with cellular aging. They are specialized DNA-protein structures at the ends of chromosome with remarkable functions-preventing their recognition as double-stranded DNA breaks, protecting their recombination and degradation, and avoiding a DNA damage cellular response. Telomere shortening is currently considered the best aging marker, but is also a predictor for age-related diseases, including cardiovascular diseases. Biological age clearly seems to be a better predictor of vascular risk rather than chronological age. This concept is supported by key assumptions that peripheral blood leukocyte telomere content accurately reflects that of the vascular wall and its decrease is associated with premature vascular disease. Thus, we are analyzing whether the mean of blood leukocyte telomere length might also be a predictor for sporadic thoracic aortic aneurysm (S-TAA). The preliminary results seem to be promising. Shorter telomeres were detected in patients than in controls. Thus, mean of blood leukocyte telomere length could contribute to identify individuals at S-TAA risk.
\end{abstract}

\section{Introduction}

T He PREVAlence of CARdiovascular diseases (CVD) rises with aging and is one of the main causes of mortality in Western countries. In view of the progressive population aging, a better understanding of age-associated diseases, including CVD, and preventive measures are needed. Among CVD, sporadic thoracic aortic aneurysm (STAA), the major disease affecting the aorta, has increased incidence particularly in the aged population, as reported by reliable epidemiological observations. ${ }^{1-4}$

S-TAA is predominantly a silent illness, until rupture or dissection occurs, and is insidious in its onset and progression. ${ }^{1-3}$ It has a complex pathophysiology that differs from that of abdominal aorta aneurysm (AAA) principally associated with atherosclerosis. ${ }^{1-3}$ Several cardiovascular risk factors (i.e., hypertension, oxidative stress, smoking, etc.) seem to explain most risk of S-TAA in the general population. ${ }^{1-3}$ However, little is known about its precise cellular and molecular mechanisms and genetic determinants. ${ }^{1-3}$ In particular, at an individual level, there is wide variation in both its occurrence and complications (i.e., dissection and rupture) and age and gender manifestation, even in individuals with the same risk factor profiles. ${ }^{1-3}$ The reasons for this wide interin- dividual variation in susceptibility are poorly understood. However, it is currently emerging that interindividual variation in CVD risk, such as S-TAA, is a result of variability in the rate of biological aging. ${ }^{5,6}$

Recent studies suggest that biological age rather than chronological age is a better predictor of vascular risk. ${ }^{6-11}$ This assumption is based on the evidence that telomere content (the TTAGGG DNA repeats at the ends of chromosomes) in circulating blood leukocytes accurately reflects the biological age of the vascular wall, as recently demonstrated by Wilson et al. ${ }^{12}$ Furthermore, a series of recent epidemiological studies have demonstrated association of the reduction of blood telomere length with several CVD, such as AAA. ${ }^{6-12}$ Patients with AAA showed a shorter leukocyte telomere length compared to controls, as verified by Atturu et al. ${ }^{13}$ In light of this evidence, our studies are aimed at determining whether the mean of blood leukocyte telomere length might be a predictor for S-TAA.

\section{Materials and Methods}

Peripheral blood samples were collected from 161 S-TAA patients and 128 age- and gender-matched controls (61 [47\%] men and 67 [53\%] women; mean age, $61.08 \pm 5.83$ years]. The

\footnotetext{
${ }^{1}$ Immunosenescence Group, Department of Pathobiology and Medical and Forensic Biotechnologies, ${ }^{2}$ Unit of Cardiac Surgery, Department of Surgery and Oncology, and ${ }^{3}$ Department of Internal Medicine and Cardiovascular Diseases, University of Palermo, Italy.
} 
clinical and demographic features of patients are summarized in Table 1. For patient selection, the 2010 guidelines, ${ }^{14}$ histopathological and phenotypic analyses, and exclusion criteria for syndromic and familial forms (e.g., Marfan and Ehler-Danlos syndromes) and autoimmune connective tissue disorders were used. The controls were in good health according to their clinical history and blood tests (complete blood cell count, erythrocyte sedimentation rate, glucose, urea nitrogen, creatinine, electrolytes, C-reactive protein, liver function tests, iron, and proteins). Concerning the major cardiac risk factors, smoking (51\%), hypertension $(31 \%)$, cardiovascular ischemic familiarity $(27 \%)$, dyslipidemia (16\%), and diabetes mellitus (13\%) were evidenced (see Table 1). Furthermore, ECHO imaging examinations confirmed absence of any aorta wall abnormalities in all controls. We selected a very homogeneous population; patients and controls belonged to same ethnic group (their parents and grandparents were born in western Sicily).

Our study received approval from local ethic committees, and all participants gave their informed consent. Data were encoded to ensure patient and control protection. All measurements were performed without knowledge about nature of material.

Genomic DNA was extracted from leukocytes of both the two cohorts and the mean terminal restriction fragment (TRF) length, a marker of telomere length, was measured by a chemiluminesence technique using TeloTAGGG Telomere Length Assay kits (Roche-Applied Science, Germany). In the present pilot study, we examined the mean TRF length in 10 patients and 10 controls selected randomly, but having the

Table 1. Demographic and Clinical Characteristics of 161 S-TAA Patients and 128 Controls

\begin{tabular}{|c|c|c|c|c|c|c|}
\hline Variables & Patients & Male & Female & Controls & $\begin{array}{l}\text { P1 (cases vs. } \\
\text { controls) }\end{array}$ & $\begin{array}{c}\text { P2 (male vs. } \\
\text { female) }\end{array}$ \\
\hline Demographic characteristics & $\mathrm{n}=161$ & $\mathrm{n}=127$ & $\mathrm{n}=34$ & $\mathrm{n}=128$ & & \\
\hline Age, mean (standard deviation, SD) & $63(10.7)$ & $63(11)$ & $64(9)$ & $61.1(5,8)$ & 0.834 & 0.594 \\
\hline Male sex, number $(\%)$ & $127(78)$ & & & $61(47)$ & & \\
\hline Female sex, number (\%) & $34(22)$ & & & $67(53)$ & & \\
\hline Body mass index, mean (SD) & $27(4.3)$ & $26.9(3.8)$ & $27.5(5.6)$ & $26.9(2.9)$ & 0.898 & 0.963 \\
\hline \multicolumn{7}{|l|}{ Size and location } \\
\hline Size (mm), mean (SD) & $53.3(8)$ & $52.9(7.5)$ & $55(9.8)$ & $0(0)$ & & 0.191 \\
\hline Location, number $(\%)$ : & & & & $0(0)$ & & 0.198 \\
\hline Ascending aorta & $81(50)$ & $20(59)$ & $61(48)$ & & & \\
\hline Aortic bulb & $18(11)$ & $1(3)$ & $17(13.4)$ & & & \\
\hline Ascending aorta and aortic bulb & $62(39)$ & $13(38)$ & $49(38.6)$ & & & \\
\hline \multicolumn{7}{|l|}{ Co-morbidity conditions, number $(\%)$} \\
\hline Aortic aneurysm familiarity & $9(5.5)$ & $8(6.2)$ & $1(0.3)$ & $0(0)$ & 0.026 & 0.736 \\
\hline Cardiovascular Ischemic familiarity & $59(36.6)$ & $48(38)$ & $11(32)$ & $34(27)$ & 0.089 & 0.7 \\
\hline Smoking & $73(45)$ & $67(53)$ & $6(18)$ & $66(51)$ & 0.351 & $<0.001$ \\
\hline Hypertension & $127(78.9)$ & $101(80)$ & $26(76)$ & $40(31)$ & $<0.001$ & 0.879 \\
\hline Dislipidemy & $37(23)$ & $30(24)$ & $7(21)$ & $20(16)$ & 0.158 & 0.886 \\
\hline Diabetes mellitus & $24(15)$ & $16(13)$ & $8(24)$ & $16(13)$ & 0.677 & 0.187 \\
\hline Renal failure & $5(3.1)$ & $4(3.1)$ & $1(2.9)$ & $0(0)$ & 0.168 & 0.621 \\
\hline Dissection & $18(11)$ & $5(15)$ & $13(10)$ & $0(0)$ & & 0.669 \\
\hline Bicuspid aortic valve disease & $27(16.8)$ & $21(16.5)$ & $6(18)$ & $0(0)$ & & 0.92 \\
\hline Aortic valve pathology, number $(\%)$ & & & & & & 0.766 \\
\hline Normal & $90(56)$ & $71(56)$ & $20(59)$ & $0(0)$ & & \\
\hline Prolapse & $21(13)$ & $17(13)$ & $3(9)$ & $0(0)$ & & \\
\hline Vascular calcium fibrosis & $50(31)$ & $39(31)$ & $11(32)$ & $0(0)$ & & \\
\hline Aortic valve dysfunction, number (\%) & & & & & & 0.91 \\
\hline Normal & $32(20)$ & $26(20.4)$ & $6(17.6)$ & $0(0)$ & & \\
\hline Faint incontinence & $29(18)$ & $22(17)$ & $7(20.4)$ & $0(0)$ & & \\
\hline Moderate incontinence & $34(21)$ & $28(22)$ & $6(17.6)$ & $0(0)$ & & \\
\hline Severe incontinence & $44(28))$ & $35(28)$ & $9(26.4)$ & $0(0)$ & & \\
\hline Faint stenosis & $1(0.6)$ & $1(0.8)$ & $0(0)$ & $0(0)$ & & \\
\hline Moderate stenosis & $2(1.2)$ & $1(0.8)$ & $1(3)$ & $0(0)$ & & \\
\hline Severe stenosis & $19(11.2)$ & $14(11)$ & $5(15)$ & $0(0)$ & & \\
\hline Atherosclerosis coronary syndrome, number (\%) & $54(33.8)$ & $45(36)$ & $9(26.5)$ & $0(0)$ & & 0.42 \\
\hline \multicolumn{7}{|l|}{ Drugs, number $(\%)$} \\
\hline Beta blockers & $62(39)$ & $47(37)$ & $15(44)$ & $0(0)$ & & \\
\hline Central $\alpha$-adrenergic agonists & $26(16)$ & $21(17)$ & $5(15)$ & $0(0)$ & & \\
\hline Sartans & $32(20)$ & $27(21)$ & $5(15)$ & $0(0)$ & & \\
\hline Calcium-channel blockers & $47(29)$ & $38(30)$ & $9(26)$ & $0(0)$ & & \\
\hline Angiotensin-converting enzyme (ACE) inhibitors & $66(41)$ & $55(43)$ & $11(32)$ & $21(16)$ & & \\
\hline Antidiabetic drugs & $19(12)$ & $13(10)$ & $6(18)$ & $16(13)$ & & \\
\hline Antiaggregant drugs & $51(32)$ & $44(34)$ & $7(21)$ & $40(31)$ & & \\
\hline Antidyslipidemic drugs & $36(22)$ & $30(24)$ & $6(18)$ & $0(0)$ & & \\
\hline Diuretics & $36(22)$ & $24(19)$ & $12(36)$ & $40(31)$ & & \\
\hline
\end{tabular}


same age and gender. Accordingly, the same number of males and females characterized both the two cohorts studied.

\section{Statistical analysis}

The difference in mean TRF length between cases and controls was analyzed using an independent sample $t$-test. The difference in mean TRF length in subjects with different risk factors was analyzed using bivariate correlation for continuous variables and an independent samples $t$-test for categorical variable. The independent effect of age, sex, and other risk factors on the mean TRF length was analyzed using a linear regression model controlling for case-control status.

\section{Results}

All patient and control features of our cases are summarized in Table 1. An elevated number of S-TAA men (127 vs. 34 women) led to comparison of all patient features according to gender. Comparisons were also detected between cases and controls (see Table 1).

In the present pilot study, we examined only 10 patients and 10 controls selected randomly, but considering the same age and gender. The evaluation of the mean TRF length in the two groups and their analysis demonstrated that the mean TRF length of S-TAA group (5.550, standard deviation $[S D]=0.605 \mathrm{kbp}$ ) was significantly lower than that observed in the control group (6.739, $\mathrm{SD}=0.485 \mathrm{kbp}$ ) (Fig. 1). A difference of $1.189 \mathrm{bp}$ was observed between cases and controls (95\% confidence interval [CI] 77-301 bp, $p=0.001$ ). There was no significant change in the mean TRF length with age in both patients and controls (6 bp increase per year, $\mathrm{SD}=4$; $p=0.16)$. There was also no significant correlation between aneurysm size and mean TRF length $(p=0.35)$. There was no significant difference in the mean TRF length between male and female patients. The difference in mean TRF length between subjects based on risk factors such as gender, smoking, hypertension, diabetes, and family history of S-TAA was evaluated. Sex as an independent risk factor did not have any significant effect on the mean TRF length $(p=0.99)$. In

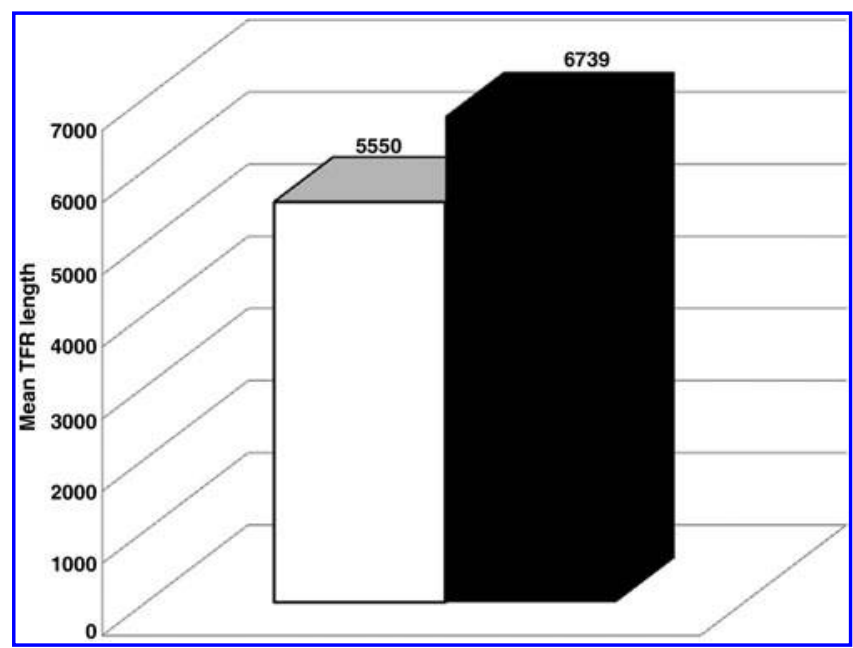

FIG. 1. The mean terminal restriction fragment (TFR) length in the two cohorts. (White bar) Sporadic thoracic aortic aneurysm (S-TAA) cases; (black bar) controls. contrast, smoking and hypertension in S-TAA cases were the only risk factors that were significantly associated with the mean TRF length $(p=0.03)$. S-TAA subjects with smoking and hypertension histories had a significantly shorter mean TRF length $(5.491, \mathrm{SD}=0.485)$ compared to subjects without these risk factors $(5.684, \mathrm{SD}=0.502)$ (mean difference $192 \mathrm{bp}$, CI 7-378 bp, $p=0.02$ ).

\section{Discussion}

A remarkable variability exists in the susceptibility, onset age, and steps of progression for CVD, including S-TAA, and this has been inadequately explained by the presence or absence of conventional risk factors. Today, it has been assumed that differences in biological aging might provide an additional and more appropriate component of the observed variability., ${ }^{5,6}$ In particular, telomere length provides a potential marker of an individual's biological age, and shorter telomeres reflect a more advanced biological age. ${ }^{5,6}$ Telomere length at birth is determined mainly by genetic factors. ${ }^{5}$ Telomere attrition occurs as a consequence of cellular replication and can be accelerated by dangerous environmental factors, such as oxidative stress and smoking, as well and is largely documented. ${ }^{6}$ When telomeres reach a critical threshold, the cell will enter senescence and become dysfunctional. ${ }^{5,6}$ Furthermore, within an individual, there is a high similarity in telomere length between various tissues. ${ }^{5,6}$ As consequence, leukocyte telomere length is commonly used as an optimal marker for overall telomere length. In particular, peripheral blood leukocyte telomere content accurately reflects that of vascular wall, and its decrease is associated with premature vascular disease. ${ }^{12}$ So, the blood leukocyte telomere DNA content may be used as a convenient surrogate for vascular aging in population studies. ${ }^{12}$

On the basis of these observations, not unexpectedly a series of epidemiological studies have demonstrated an association between blood telomere length and aging-related diseases, such as CVD. Shorter telomeres have been demonstrated in subjects with several CVD pathologies, including chronic heart failure, atherosclerosis, ventricular dysfunction, coronary artery calcification, and aortic valve stenosis. ${ }^{6-12}$ Furthermore, telomere length has been also identified as an independent predictor of heart disease-related events. ${ }^{6-12}$ In addition, a significant reduction in telomere content of AAA wall samples compared to normal aorta and in AAA patients than controls has been detected. ${ }^{12,13}$

The mechanisms linking blood telomere attrition to CVD have been studied recently, ${ }^{6}$ and number of potential explanations have been suggested. ${ }^{6}$ First, the strong association between human blood leukocyte and vessel wall telomere content may be determined genetically, and shorter telomeres may predispose to CVD development. ${ }^{6}$ In contrast, it has been assumed that the shorter telomeres in individuals with CVD may be acquired due to accelerated telomere attrition in the blood leukocyte and vessel wall occurring in tandem following exposure to CVD risk factors, such as hypertension, diabetes, dyslipidemia, obesity, and smoking. ${ }^{6}$ Chronic inflammation and oxidative damage to DNA are probable common mechanisms for the latter hypothesis. ${ }^{6}$ On the other hand, we have particularly outlined in our studies the key role of inflammation in CVD pathophysiology. ${ }^{15,16}$ Accordingly, it is plausible that the actual length of the 
telomere in the vessel wall has less to do with the process of aging and is simply the result of an inflammatory process that shortens telomeres in parallel in many tissues. ${ }^{6}$

In the light of these observations, we examined whether the mean of blood leukocyte telomere length might be a predictor for S-TAA. The preliminary results obtained are promising and show that S-TAA patients have significantly shorter leukocyte TRF length compared to controls. Furthermore, the assessment of biological effects of S-TAA risk factors on leukocyte TRF length showed that smoking and hypertension were the only risk factors significantly associated with reduction of the mean TRF length $(p=0.03)$. Their association with a reduction of mean TRF length seems plausible with the biological effects that they mediate on the aortic wall. ${ }^{6}$

Thus, our results, although based on a limited number of cases and controls, seem to suggest that the mean of blood leukocyte telomere length might be a predictor for S-TAA. However, larger studies are needed to confirm and validate our preliminary data. On the other hand, S-TAA is a welldefined phenotype, and, consequently, we did not find difficulty in identifying patients with this aorta wall disease. Furthermore, we have measured blood telomere length using terminal restriction fragment assay (Southern blot technique) rather than the more recently developed assays like polymerase chain reaction or quantitative hybridization. Although the terminal restriction fragment assay is time consuming and laborious, it measures the telomere length directly rather than measuring the telomere content. Thus, these experimental conditions might assure obtaining very interesting data.

In conclusion, telomere assay could contribute to identify individuals at risk for S-TAA. In addition, our results seem to suggest that vascular biological aging might have a strong role in the S-TAA pathogenesis. If it can be proved that telomeres are not only associated but also causally involved in the pathogenesis of S-TAA, this result might provide exciting new avenues for the development of future preventive and therapeutic strategies.

\section{Acknowledgment}

This work was supported by grants from the Italian Ministry of Education, University and Research to G.C.

\section{Author Disclosure Statement}

No competing financial interests exist.

\section{References}

1. Ince $\mathrm{H}$, Nienaber CA. Etiology, pathogenesis and management of thoracic aortic aneurysm. Nat Clin Pract Cardiovasc Med 2007;4:418-427.

2. El-Hamamsy I, Yacoub MH. Cellular and molecular mechanisms of thoracic aortic aneurysms. Nat Rev Cardiol 2009;6: 771-786.

3. Elefteriades JA, Farkas EA. Thoracic aortic aneurysm clinically pertinent controversies and uncertainties. J Am Coll Cardiol 2010;55:841-857.

4. National Center for Injury Prevention and Control. WISQARS Leading Causes of Death Reports, 1999-2006. Available at:http://webappa.cdc.gov/sasweb/ncipc/leadcaus10.html/.

5. Zhu H, Belcher M, van der Harst P. Healthy aging and disease: Role for telomere biology? ClinSci (Lond) 2011;120:427-440.
6. De Meyer T, Rietzschel ER, De Buyzere ML, Van Criekinge $\mathrm{W}$, Bekaert $\mathrm{S}$. Telomere length and cardiovascular aging: the means to the ends? Ageing Res Rev 2011;10:297-303.

7. Epel ES, Merkin SS, Cawthon R, Blackburn EH, Adler NE, Pletcher MJ, Seeman TE. The rate of leukocyte telomere shortening predicts mortality from cardiovascular disease in elderly men. Aging (Albany NY) 2008;1:81-88.

8. Mainous AG, Diaz VA. Telomere length as a risk marker for cardiovascular disease: The next big thing? Expert Rev Mol Diagn2010;10:969-971.

9. Huzen J, de Boer RA, van Veldhuisen DJ, van Gilst WH, van der Harst P. The emerging role of telomere biology in cardiovascular disease. Front Biosci 2010;15:35-45.

10. Saliques S, Zeller M, Lorin J, Lorgis L, Teyssier JR, Cottin Y, Rochette L, Vergely C. Telomere length and cardiovascular disease. Arch Cardiovasc Dis 2010;103:454-459.

11. Fitzpatrick AL, Kronmal RA, Kimura M, Gardner JP, Psaty BM, Jenny NS, Tracy RP, Hardikar S, Aviv A. Leukocyte telomere length and mortality in the Cardiovascular Health Study. I Gerontol A Biol Sci Med Sci 2011; 66:421-429.

12. Wilson WR, Herbert KE, Mistry $Y$, Stevens SE, Patel HR, Hastings RA, Thompson MM, Williams B. Blood leucocyte telomere DNA content predicts vascular telomere DNA content in humans with and without vascular disease. Eur Heart J 2008;29:2689-2694.

13. Atturu G, Brouilette S, Samani NJ, London NJ, Sayers RD, Bown MJ. Short leukocyte telomere length is associated with abdominal aortic aneurysm (AAA). Eur J Vasc Endovasc Surg 2010;39:559-564.

14. Hiratzka LF, Bakris GL, Beckman JA, Bersin RM, Carr VF, Casey DE Jr, et al. ACCF/AHA/AATS/ACR/ASA/SCA/ SCAI/SIR/STS/SVM guidelines for the diagnosis and management of patients with Thoracic Aortic Disease: A report of the American College of Cardiology Foundation/ American Heart Association Task Force on Practice Guidelines, American Association for Thoracic Surgery, American College of Radiology, American Stroke Association, Society of Cardiovascular Anesthesiologists, Society for Cardiovascular Angiography and Interventions, Society of Interventional Radiology, Society of Thoracic Surgeons, and Society for Vascular Medicine. J Am Coll Cardiol 2010;55: e27-e129.

15. Licastro F, Candore G, Lio D, Porcellini E, Colonna-Romano G, Franceschi C, Caruso C. Innate immunity and inflammation in ageing: A key for understanding age-related diseases. Immun Ageing 2005;2:8.

16. Candore G, Balistreri CR, Caruso M, Grimaldi MP, Incalcaterra E, Listì F, Vasto S, Caruso C. Pharmacogenomics: a tool to prevent and cure coronary heart disease. Curr Pharm Des 2007;13:3726-3734.

Address correspondence to: Carmela Rita Balistreri

Department of Pathobiology and Medical and Forensic Biotechnologies University of Palermo

Corso Tukory 211

Palermo, 90134

Italy

E-mail: carmelarita.balistreri@unipa.it 